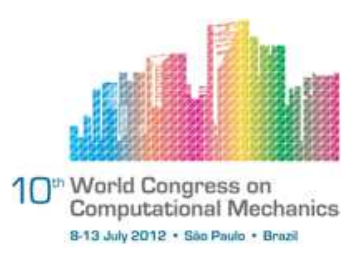

\title{
NUMERICAL ANALYSIS OF A LOCALLY PROJECTED DISCONTINUOUS GALERKIN METHOD FOR ELLIPTIC PROBLEMS
}

\author{
N. C. B. Arruda, A. F. D. Loula, R. C. Almeida \\ Laboratório Nacional de Computação Científica, (nataliac@ lncc.br)
}

\begin{abstract}
In this paper we study a new discontinuous Galerkin method which uses a computational structure compatible with conforming finite element methods, reducing considerably the number of degrees-of-freedom. The Locally Discontinuous but Globally Continuous Galerkin method starts with a discontinuous finite element space and constructs a continuous representation for it by means of local projections. The used technique is similar to that employed in hybridizable methods, and discontinuous solution is recovered by solving local element-wise problems. We present the numerical analysis of the method and numerical results to confirm the predicted convergence rates. Moreover, numerical experiments are conducted in order to evaluate the better performance of this formulation when compared to the continuous or discontinuous Galerkin formulations.
\end{abstract}

Keywords: discontinuous Galerkin, hybridizable discontinuous Galerkin.

\section{INTRODUCTION}

A great variety of Discontinuous Galerkin (DG) methods have been proposed and analyzed over the last decades for elliptic [7, 15, 3, 4, 14, 16, 27, 8, 26, 5], parabolic [2, 25] and hyperbolic $[24,22,18,19,1,20,21]$ problems. Robustness, flexibility for implementing $h$ and $p$-adaptivity strategies and easy parallelization are well known advantages of DG methods arising from the use of broken finite element spaces. However, the practical utility of DG methods has been limited by their complexity of formulation and computational implementation. Moreover, the use of DG methods requires a much larger number of degrees-of-freedom, when compared with classical continuous Galerkin methods.

Recent works have been presented some alternatives to reduce computational cost of DG methods. A Multiscale Discontinuous Galerkin Method (MDG) with the computational structure and cost of a conforming method was introduced by Hughes, Scovazzi, Bochev and Buffa in [17] and analyzed by Buffa, Hughes and Sangalli in [9]. The MDG formulation uses local, element-wise problems to project a continuous finite element space into a given discontinuous space, and then applies a discontinuous Galerkin formulation. Another approach was derived from a combination between DG formulations and hybrid methods, the hybridizable discontinuous Galerkin (HDG) methods [13, 10, 11, 12,23]. These methods reduce computational cost, with improved stability but keeping the robustness and flexibility of DG methods. 
Based on the ideas supporting hybridization techniques and the MDG method we propose in [5] the locally discontinuous but globally continuous finite element method (LDGC). This method combines the advantages of Discontinuous Galerkin methods with the element based data structure and reduced computational cost of classical conforming finite element methods. The proposed method can be viewed as a stabilized hybrid formulation consisting of locally discontinuous Galerkin problems coupled to a globally continuous problem. In [5] the LDGC method is presented and analyzed for Poisson problem, according to DG framework studied in [4]. In this paper we present the LDGC method for Reaction-Diffusion problems, and the main results of the numerical analysis, following the approach presented in [25].

The LDGC method is tested on simple elliptic problems with known exact solutions. Convergence studies confirm the optimal rates of convergence predicted by the numerical analysis. In all examples analyzed the LDGC approximations exhibited accuracy equivalent or even better than the corresponding DG approximations. The remainder of the paper is organized as follows. Section 2 is dedicated to a summary of the main features of DG formulations for reaction-diffusion problems. The LDGC method is introduced in Section 3. A numerical analysis of the LDGC formulation is presented in Section 4 showing that it preserves the main properties of the associated DG method. Numerical results are presented in Section 5 and some concluding remarks are drawn in Section 6.

\section{DG METHODS}

We consider the second order elliptic model problem

$$
\begin{aligned}
-\varepsilon \Delta u+\sigma u & =f \text { in } \Omega, \\
u & =g \text { on } \Gamma
\end{aligned}
$$

where $\Omega \subset \mathbb{R}^{d}(d=2)$ is an open bounded domain with a Lipschitz boundary $\Gamma=\partial \Omega, \Delta$ is the Laplacian operator, $\varepsilon>0$ is the diffusion coefficient, $\sigma>0$ is the reaction coefficient, the source term $f \in L^{2}(\Omega)$ and the Dirichlet boundary condition $g \in H^{1 / 2}(\Gamma)$.

The notation commonly utilized in DG methods is presented, since is also adopted in LDGC method, which will be introduced in next section. Let

$$
\mathcal{T}_{h}=\{\mathcal{K}\}:=\text { union of all elements } K
$$

be a regular finite element partition of the two dimension domain $\Omega$ and let

$$
\mathcal{E}_{h}=\left\{e: e \text { is an edge of } K \text { for all } K \in \mathcal{T}_{h}\right\}
$$

denote the set of all edges of all elements $K$ of the mesh $\mathcal{T}_{h}$,

$$
\mathcal{E}_{h}^{0}=\left\{e \in \mathcal{E}_{h} e \text { is an interior edge }\right\}
$$

the set of interior edges, and

$$
\mathcal{E}_{h}^{\partial}=\mathcal{E}_{h} \cap \partial \Omega,
$$


the set of edges of $\mathcal{E}_{h}$ on the boundary of $\Omega$. Discontinuous Galerkin methods are normally formulated and analyzed using well defined averages and jumps on interior edges, for a scalar function $\varphi$

$$
\{\varphi\}=\frac{1}{2}\left(\varphi_{1}+\varphi_{2}\right), \quad \llbracket \varphi \rrbracket=\varphi_{1} \mathbf{n}_{1}+\varphi_{2} \mathbf{n}_{2} \quad \text { on } e \in \mathcal{E}_{h}^{0}
$$

and for a vector field $\tau$

$$
\{\boldsymbol{\tau}\}=\frac{1}{2}\left(\boldsymbol{\tau}_{1}+\boldsymbol{\tau}_{2}\right), \quad \llbracket \boldsymbol{\tau} \rrbracket=\boldsymbol{\tau}_{1} \cdot \mathbf{n}_{1}+\boldsymbol{\tau}_{2} \cdot \mathbf{n}_{2} \quad \text { on } e \in \mathcal{E}_{h}^{0} .
$$

For boundary edges it is usually set

$$
\{\varphi\}=\varphi, \quad \llbracket \varphi \rrbracket=\varphi \mathbf{n}, \quad\{\boldsymbol{\tau}\}=\boldsymbol{\tau}, \quad \llbracket \boldsymbol{\tau} \rrbracket=\boldsymbol{\tau} \cdot \mathbf{n} \quad \text { on } e \in \mathcal{E}_{h}^{\partial} .
$$

Let $V_{h}$ denote the broken function space

$$
V_{h}=\left\{v \in L^{2}(\Omega):\left.v\right|_{K} \in S_{k}(K) \quad \forall K \in \mathcal{T}_{h}\right\}
$$

where $S_{k}(K)=P_{k}(K)$ (the space of polynomial functions of degree at most $k$ in both variables) or $S_{k}(K)=Q_{k}(K)$ (the space of polynomial functions of degree at most $k$ in any variable), equipped with the broken Sobolev norm:

$$
\|\| v \|_{H^{s}\left(\mathcal{K}_{h}\right)}:=\left(\sum_{K \in \mathcal{K}_{h}}\|v\|_{H^{s}(K)}^{2}\right)^{1 / 2}
$$

A well established class of primal Discontinuous Galerkin (DG) methods for our model problem is formulated as follows: find $u_{h} \in V_{h}$ such that

$$
a_{h}\left(u_{h}, v_{h}\right)=f_{h}\left(v_{h}\right) \quad \forall v \in V_{h}
$$

with

$$
\begin{aligned}
a_{h}\left(u_{h}, v_{h}\right) & =\sum_{K \in \mathcal{T}_{h}} \int_{K}\left(\varepsilon \nabla u_{h} \cdot \nabla v_{h}+\sigma u_{h} v_{h}\right) d x \\
& +\sum_{e \in \mathcal{E}_{h}} \int_{e}\left(s \llbracket u_{h} \rrbracket \cdot\left\{\varepsilon \nabla v_{h}\right\}-\llbracket v_{h} \rrbracket \cdot\left\{\varepsilon \nabla u_{h}\right\}\right) d s \\
& +\sum_{e \in \mathcal{E}_{h}^{0}} \eta \int_{e} \llbracket u_{h} \rrbracket \cdot \llbracket v_{h} \rrbracket d s+\sum_{e \in \mathcal{E}_{h}^{\partial}} \eta \int_{e} u_{h} v_{h} d s
\end{aligned}
$$

and

$$
f_{h}(v)=\sum_{K \in \mathcal{T}_{h}} \int_{K} f v d x+\int_{\partial \Omega}(\varepsilon \nabla v \cdot \mathbf{n}) g d s+\eta \int_{\partial \Omega} g v d s .
$$

where the constant $s$ usually has been chosen -1 (SIPG), 0 (IIPG) or 1 (NIPG) (see [27] for more details). The penalty function $\eta$ is defined as

$$
\eta=\frac{\eta_{0}}{h}, \quad \forall e \in \mathcal{E}_{h}
$$


with $\eta_{0}>0$. The numerical analysis of this class discontinuous Galerkin methods fits in the framework for the analysis of DG methods provided in reference [25]. See also references $[4,2,8]$. Since the domain $\Omega$ is polygonal, there exists $\alpha>0$ such that $h \leq \alpha h_{e}$, where $h_{e}$ is the measure of the edge $e \in \partial K$. For the sake of simplicity we will present all estimates in terms of $h$. We consider the following energy norm

$$
\|v\|_{\mathcal{E}}^{2}:=\sum_{K \in \mathcal{K}_{h}} \int_{K} \varepsilon \nabla v \cdot \nabla v d x+\int_{\Omega} \sigma v^{2}+\sum_{e \in \mathcal{E}_{h}} \eta \int_{e} \llbracket v \rrbracket \cdot \llbracket v \rrbracket d s ;
$$

to present a summary of the main results on the numerical analysis of the above DG formulation based on references $[4,25]$.

DG Consistency. The solution $u$ the model problem (1) - (2) satisfies

$$
a_{h}\left(u, v_{h}\right)=f_{h}\left(v_{h}\right) \quad \forall v_{h} \in V_{h} .
$$

DG Local conservation. Fixing an element $K$ belonging to the interior of the domain and choosing $v_{h}=1$ in $K, v_{h}=0$ elsewhere DG method (11) reduces to

$$
\int_{K}\left(\sigma u_{h}-f\right) d x+\sum_{e \in \partial K} \eta \int_{e} \llbracket u_{h} \rrbracket \cdot \mathbf{n} d s=\sum_{e \in \partial K} \int_{e}\left\{\varepsilon \nabla u_{h}\right\} \cdot \mathbf{n} d s,
$$

which expresses the local conservation of the DG formulation with boundary stabilization.

DG Stability. For $\eta_{0}$ sufficiently large there exists a constant $\alpha_{s}>0$, independent of $s$ and $h$, such that

$$
a_{h}\left(v_{h}, v_{h}\right) \geq \kappa\left\|v_{h}\right\|_{\mathcal{E}}^{2} \quad \forall v_{h} \in V_{h} .
$$

DG Continuity. If $\eta_{0}>0$ for all $e$, there exists a constant $\alpha_{b}$ such that

$$
a_{h}\left(u_{h}, v_{h}\right) \leq M\left\|u_{h}\right\|_{\mathcal{E}}\left\|v_{h}\right\|_{\mathcal{E}} \quad \forall u_{h}, v_{h} \in V_{h}
$$

DG Approximation. For any $u \in H^{k+1}(\Omega)$ the continuous interpolant of $u, u_{I} \in V_{h}$, satisfies

$$
\|\left.\left|u-u_{I}\right|\right|_{H^{q}\left(\mathcal{K}_{h}\right)} \leq C h^{\min (k+1, s)-q}|u|_{H^{s}(K)} .
$$

DG Error estimate in the energy norm. Consistency, stability and continuity lead to the estimate

$$
\left\|u-u_{h}\right\|_{\mathcal{E}} \leq C h^{\min (k+1, r)-1}\left|\|u \mid\|_{H^{r}\left(\mathcal{T}_{h}\right)} .\right.
$$

with $r=3 / 2$.

DG Error estimate in $L^{2}(\Omega)$. The adjoint consistent DG formulation corresponding to $s=-1$ presents also the optimal rate of convergence in $L^{2}(\Omega)$ norm

$$
\left\|u-u_{h}\right\|_{0, \Omega} \leq C h^{\min (k+1, r)} \mid\|u\|_{H^{r}\left(\mathcal{T}_{h}\right)} .
$$

In the next section we introduce a new formulation preserving the all these above properties of DG methods but with implementation and computational cost equivalent to a continuous Galerkin method. 


\section{THE LDGC METHOD}

To define the Discontinuous Galerkin formulation based on a local projection we introduce a new variable $\lambda$, uniquely defined as $\lambda=\left.u\right|_{e}$ on each edge $e \in \mathcal{E}_{h}$, and consider the finite dimension function sets

$$
\begin{aligned}
& M_{h}^{g}=\left\{\lambda \in C^{0}\left(\mathcal{E}_{h}\right):\left.\lambda\right|_{e}=P_{l}(e), \forall e \in \mathcal{E}_{h}^{0},\left.\lambda\right|_{e}=g, \forall e \in \mathcal{E}_{h}^{\partial}\right\}, \\
& M_{h}=\left\{\lambda \in C^{0}\left(\mathcal{E}_{h}\right):\left.\lambda\right|_{e}=P_{l}(e), \forall e \in \mathcal{E}_{h}^{0},\left.\lambda\right|_{e}=0, \forall e \in \mathcal{E}_{h}^{\partial}\right\},
\end{aligned}
$$

where $P_{l}(e)$ is the space of of polynomials of degree at most $l$ on each edge $e$. The Locally Discontinuous but Globally Continuous Galerkin method is formulated as:

Find the pair $\left\{u_{h}, \lambda_{h}\right\} \in V_{h} \times M_{h}^{g}$ such that, for all $\left\{v_{h}, \mu_{h}\right\} \in V_{h} \times M_{h}$,

$$
\begin{aligned}
& \sum_{K \in \mathcal{T}_{h}} \int_{K} \varepsilon \nabla u_{h} \cdot \nabla v_{h}+\sigma u_{h} v_{h} d x-\int_{\partial K}\left(\varepsilon \nabla u_{h} \cdot \mathbf{n}\right)\left(v_{h}-\mu_{h}\right) d s \\
& \quad+s \int_{\partial K}\left(\varepsilon \nabla v_{h} \cdot \mathbf{n}\right)\left(u_{h}-\lambda_{h}\right) d s+\int_{\partial K} \beta\left(u_{h}-\lambda_{h}\right)\left(v_{h}-\mu_{h}\right) d s=\int_{K} f v_{h} d x
\end{aligned}
$$

with $s$ as defined before. The penalty function $\beta$ is such that

$$
\beta=\frac{\beta_{0}}{h}
$$

with $\beta_{0}>0$. Given that $v_{h}$, belonging to the broken function space $V_{h}$, is defined independently on each element $K \in \mathcal{T}_{h}$, we observe that equation (23) can be split into a set of local problems defined on each element $K$ and a global problem defined on $\mathcal{E}_{h}$. Consequently, the LDGC method can be presented as:

Find $\left.u_{h}\right|_{K} \in V_{h}(K)=\left.V_{h}\right|_{K}$ and $\lambda_{h} \in M_{h}^{g}$, such that

$$
\begin{aligned}
\int_{K}\left(\varepsilon \nabla u_{h} \cdot \nabla v_{h}+\sigma u_{h} v_{h}\right) d x & -\int_{\partial K}\left(\varepsilon \nabla u_{h} \cdot \mathbf{n}\right) v_{h} d s+s \int_{\partial K}\left(\varepsilon \nabla v_{h} \cdot \mathbf{n}\right)\left(u_{h}-\lambda_{h}\right) d s \\
+\int_{\partial K} \beta\left(u_{h}-\lambda_{h}\right) v_{h} d s & =\int_{K} f v_{h} d x,\left.\quad v_{h}\right|_{K} \in V_{h}(K)
\end{aligned}
$$

and

$$
\sum_{K \in \mathcal{T}_{h}} \int_{\partial K}\left(\varepsilon \nabla u_{h} \cdot \mathbf{n}\right) \mu_{h} d s+\int_{\partial K} \beta\left(\lambda_{h}-u_{h}\right) \mu_{h} d s=0, \quad \forall \mu_{h} \in M_{h} .
$$

\subsection{The local problems}

Equation (25) can be solved locally to find $u$ as a function of $\lambda$. Moreover, we define the local operators $a_{K}(u, v)$ and $b_{K}(\lambda, v)$, and the linear functional $f_{K}(v)$, such that

$$
\begin{aligned}
a_{K}\left(u_{h}, v_{h}\right) & =\int_{K}\left(\varepsilon \nabla u_{h} \cdot \nabla v_{h}+\sigma u_{h} v_{h}\right) d x \\
& +s \int_{\partial K} u_{h}\left(\varepsilon \nabla v_{h} \cdot \mathbf{n}\right) d s-\int_{\partial K} v_{h}\left(\varepsilon \nabla u_{h} \cdot \mathbf{n}\right) d s+\beta \int_{\partial K} u_{h} v_{h} d s \\
b_{K}\left(\lambda_{h}, v_{h}\right) & =s \int_{\partial K} \lambda_{h}\left(\varepsilon \nabla v_{h} \cdot \mathbf{n}\right) d s-\beta \int_{\partial K} \lambda v_{h} d s \\
f_{K}\left(v_{h}\right) & =\int_{K} f v_{h} d x
\end{aligned}
$$


Then, the local problem is given by:

For any given $\lambda_{h}$, find $\left.u_{h}\right|_{K} \in S_{k}(K)$ such that

$$
a_{K}\left(u_{h}, v_{h}\right)+b_{K}\left(\lambda_{h}, v_{h}\right)=f_{K}\left(v_{h}\right), \quad \forall v_{h} \in S_{k}(K) .
$$

Considering $\mathbf{A}_{K}$ and $\mathbf{B}_{K}$ the matrices generated by the local operators $a_{K}(\cdot, \cdot)$ and $b_{K}(\cdot, \cdot)$, respectively, and $\mathbf{F}_{K}$ the vector originating from $f_{K}(\cdot)$, we can rewrite the local problem (30) in a matrix form, expressing $\mathbf{u}$ in terms of $\boldsymbol{\lambda}$ :

$$
\mathbf{A}_{K} \mathbf{u}+\mathbf{B}_{K} \boldsymbol{\lambda}=\mathbf{F}_{K}
$$

Given that $\mathbf{A}_{K}$ is positive definite, we solve the system (31) to obtain

$$
\mathbf{u}=\mathbf{A}_{K}^{-1}\left(\mathbf{F}_{K}-\mathbf{B}_{K} \boldsymbol{\lambda}\right) .
$$

\subsection{The global problem}

Based on equation (26), we define the local operators $c_{K}(u, \mu)$ and $d_{K}(\lambda, \mu)$, such that

$$
\begin{aligned}
c_{K}(u, \mu) & =\int_{\partial K} \mu(\varepsilon \nabla u \cdot \mathbf{n}) d s-\beta \int_{\partial K} u \mu d s ; \\
d_{K}(\lambda, \mu) & =\beta \int_{\partial K} \lambda \mu d s .
\end{aligned}
$$

Considering $\mathbf{C}_{K}$ and $\mathbf{D}_{K}$ the matrices generated by the local operators $c_{K}(\cdot, \cdot)$ and $d_{K}(\cdot, \cdot)$, respectively, we can rewrite equation (26) in a matrix form:

$$
\sum_{K \in \mathcal{T}_{h}} \mathbf{C}_{K} \mathbf{u}+\mathbf{D}_{K} \boldsymbol{\lambda}=0
$$

Replacing (32) in (35), we obtain

$$
\sum_{K \in \mathcal{T}_{h}} \mathbf{C}_{K} \mathbf{A}_{K}^{-1}\left(\mathbf{F}_{K}-\mathbf{B}_{K} \boldsymbol{\lambda}\right)+\mathbf{D}_{K} \boldsymbol{\lambda}=0
$$

which gives

$$
\sum_{K \in \mathcal{T}_{h}}\left(\mathbf{D}_{K}-\mathbf{C}_{K} \mathbf{A}_{K}^{-1} \mathbf{B}_{K}\right) \boldsymbol{\lambda}=\sum_{K \in \mathcal{T}_{h}}-\mathbf{C}_{K} \mathbf{A}_{K}^{-1} \mathbf{F}_{K}
$$

We can consider equation (32) under another point of view, defining an affine operator $\mathcal{H}: M_{h} \times L^{2}(K) \rightarrow V_{h}$, which associates to each $\left(M_{h}, f\right) \in M_{h}^{0} \times L^{2}(\Omega)$ a discontinuous solution $u_{h} \in V_{h}$. Thus, equation (32) can be rewritten as $u_{h}=\mathcal{H}\left(\lambda_{h}, f\right)$. Therefore, the LDGC global formulation is given by: find $\lambda_{h} \in \Lambda_{h}$ such that

$$
A_{L D G C}\left(\lambda_{h}, \mu_{h}\right)=0, \quad \forall \mu_{h} \in M_{h},
$$

where

$$
A_{L D G C}\left(\lambda_{h}, \mu_{h}\right)=\sum_{K \in \mathcal{T}_{h}}\left(c_{K}\left(\mathcal{H}\left(\lambda_{h}, f\right), \mu_{h}\right)+d_{K}\left(\lambda_{h}, \mu_{h}\right)\right) .
$$

Thus, since $\lambda_{h}$ is continuous, the global problem is solved by using (38), which reduces strongly the number of degrees-of-freedom. The discontinuous solution is recovered 
by solving the local element-wise problems described in (30), using equation (31) element by element. An interesting issue of this method is that different degrees of the interpolation polynomials can be used for $u_{h}$ and $\lambda_{h}$. Considering $k$ and $l$ the degrees of the interpolation polynomials for $u_{h}$ and $\lambda_{h}$, respectively, the choice $k>l$ produces better approximations for the discontinuous solution $u_{h}$. This selection keeps the number of degrees-of-freedom reduced and has almost the same computational cost of the alternative $k=l$.

\subsection{Main Properties of LDGC Method}

Now we present some properties of LDGC method that are preserved from DG methods, like consistency and local conservation. We start rewriting the LDGC method in a DG framework, giving rise a coupled problem where these properties can be obtained naturally. Using identity

$$
\sum_{K \in \mathcal{T}_{h}} \int_{\partial K}(\boldsymbol{\tau} \cdot \mathbf{n}) \varphi d s=\sum_{e \in \mathcal{E}_{h}} \int_{e}\{\boldsymbol{\tau}\} \cdot \llbracket \varphi \rrbracket d s+\sum_{e \in \mathcal{E}_{h}^{0}} \int_{e}\{\varphi\} \llbracket \boldsymbol{\tau} \rrbracket d s,
$$

considering that $\lambda_{h}$ and $\mu_{h}$ are uniquely defined on $\mathcal{E}_{h}$ and taking into account the following identities:

$$
\begin{aligned}
\varphi_{1} & =\frac{1}{2}\left(\varphi_{1}-\varphi_{2}\right)+\frac{1}{2}\left(\varphi_{1}+\varphi_{2}\right), \\
\varphi_{2} & =\frac{1}{2}\left(\varphi_{1}+\varphi_{2}\right)-\frac{1}{2}\left(\varphi_{1}-\varphi_{2}\right),
\end{aligned}
$$

the LDGC method can be presented as:

Find the pair $\left\{u_{h}, \lambda_{h}\right\} \in V_{h} \times M_{h}$ such that

$$
A\left(\left\{u_{h}, \lambda_{h}\right\},\left\{v_{h}, \mu_{h}\right\}\right)=F\left(\left\{v_{h}, \mu_{h}\right\}\right) \quad \forall\left\{v_{h}, \mu_{h}\right\} \in V_{h} \times M_{h}
$$

where

$$
\begin{aligned}
A\left(\left\{u_{h}, \lambda_{h}\right\},\left\{v_{h}, \mu_{h}\right\}\right) & =\sum_{K \in \mathcal{T}_{h}} \int_{K} \varepsilon \nabla u_{h} \cdot \nabla v_{h}+\sigma u_{h} v_{h} d x \\
& +\sum_{e \in \mathcal{E}_{h}} \int_{e}\left(s \llbracket u_{h} \rrbracket \cdot\left\{\varepsilon \nabla v_{h}\right\}-\llbracket v_{h} \rrbracket \cdot\left\{\varepsilon \nabla u_{h}\right\}\right) d s \\
& +\sum_{e \in \mathcal{E}_{h}^{0}} \int_{e} \frac{\beta}{2} \llbracket u_{h} \rrbracket \cdot \llbracket v_{h} \rrbracket d s+\sum_{e \in \mathcal{E}_{h}^{\partial}} \int_{e} \beta u_{h} v_{h} d s \\
& +\sum_{e \in \mathcal{E}_{h}^{0}} \int_{e}\left(\llbracket \varepsilon \nabla u_{h} \rrbracket\left(\mu_{h}-\left\{v_{h}\right\}\right)-s \llbracket \varepsilon \nabla v_{h} \rrbracket\left(\lambda_{h}-\left\{u_{h}\right\}\right)\right) d s \\
& +\sum_{e \in \mathcal{E}_{h}^{0}} \int_{e} 2 \beta\left(\lambda_{h}-\left\{u_{h}\right\}\right)\left(\mu_{h}-\left\{v_{h}\right\}\right) d s
\end{aligned}
$$

and

$$
F(\{v, \mu\})=f_{h}\left(v_{h}\right)
$$


with $f_{h}\left(v_{h}\right)$ given by (13).

In the above LDGC formulation (42) the boundary condition $u=g$ on $\Gamma=\partial \Omega$ is weakly imposed using the same Nitsche's approach adopted in the associated DG method (11). For this reason the unknown $\lambda_{h}$ is restricted to $M_{h}$ and identify with $g$ on the boundary $\Gamma\left(\lambda_{h}=g\right.$ on each $\left.e \in \mathcal{E}_{h}^{\partial}\right)$.

Remark 3.1. We can see the LDGC formulation (42) as a small modification of the Discontinuous Galerkin methods (11), but LDGC is not a hybridization of the associated DG formulation.

LDGC Consistency. The pair $\{u, \lambda\}$, with $u$ solution of the model problem (1) - (2) and $\lambda=\left.u\right|_{e}$ on each edge $e \in \mathcal{E}_{h}$ satisfies

$$
\begin{aligned}
\sum_{K \in \mathcal{T}_{h}} \int_{K} \varepsilon \nabla u \cdot \nabla v_{h}+\sigma u v_{h} d x & -\int_{\partial K}(\varepsilon \nabla u \cdot \mathbf{n})\left(v_{h}-\mu_{h}\right) d s+s \int_{\partial K}\left(\varepsilon \nabla v_{h} \cdot \mathbf{n}\right)(u-\lambda) d s \\
& +\int_{\partial K} \beta(u-\lambda)\left(v_{h}-\mu_{h}\right) d s=\int_{K} f v_{h} d s
\end{aligned}
$$

for all $\left\{v_{h}, \mu_{h}\right\} \in V_{h} \times M_{h}$. Considering equations (43-44), LDGC consistency (45) can be presented as

$$
\begin{aligned}
a_{h}\left(u, v_{h}\right) & -f_{h}\left(v_{h}\right) \\
& -\sum_{e \in \mathcal{E}_{h}^{0}} \int_{e} \frac{\beta}{2} \llbracket u \rrbracket \cdot \llbracket v_{h} \rrbracket d s \\
& +\sum_{e \in \mathcal{E}_{h}^{0}} \int_{e}\left(\llbracket \varepsilon \nabla u \rrbracket\left(\mu_{h}-\left\{v_{h}\right\}\right)-s \llbracket \varepsilon \nabla v_{h} \rrbracket(\lambda-\{u\})\right) d s \\
& +\sum_{e \in \mathcal{E}_{h}^{0}} \int_{e} \frac{2 \beta_{0}}{h}(\lambda-\{u\})\left(\mu_{h}-\left\{v_{h}\right\}\right) d s=0, \quad \forall\left\{v_{h}, \mu_{h}\right\} \in V_{h} \times M_{h} .
\end{aligned}
$$

Given that $u \in H^{2}(\Omega)$, then $\lambda=\left.\{u\}\right|_{e}=\left.u\right|_{e}$ and considering that $\llbracket u \rrbracket_{e}=0$ and $\llbracket \varepsilon \nabla u \rrbracket_{e}=$ $0 \forall e \in \mathcal{E}_{h}^{0}$, the consistency expression of the LDGC formulation reduces to

$$
a_{h}\left(u, v_{h}\right)-f_{h}\left(v_{h}\right)=0 \quad \forall v_{h} \in V_{h},
$$

which shows that the consistency of the LDGC formulation depends only on the consistency of the associated DG formulation.

LDGC Local conservation. Fixing an element $K$ belonging to the interior of the domain and choosing $v_{h}=1$ in $K, v_{h}=0$ elsewhere and $\mu_{h}=0$, the LDGC method (42) reduces to

$$
\begin{gathered}
\int_{K}\left(\sigma u_{h}-f\right) d x-\sum_{e \in \partial K} \int_{e}\left(\left\{\varepsilon \nabla u_{h}\right\} \cdot \mathbf{n}+\frac{1}{2} \llbracket \varepsilon \nabla u_{h} \rrbracket\right) d s \\
+\sum_{e \in \partial K} \int_{e} \beta\left(\frac{1}{2} \llbracket u_{h} \rrbracket \cdot \mathbf{n}+\left(\left\{u_{h}\right\}-\lambda_{h}\right)\right) d s=0
\end{gathered}
$$


or, equivalently

$$
\int_{K}(\sigma u-f) d x-\sum_{e \in \partial K} \int_{e} \nabla u_{h} \cdot \mathbf{n} d s+\sum_{e \in \partial K} \int_{e} \beta\left(u_{h}-\lambda_{h}\right) d s=0,
$$

which is similar to the expression for the local conservation of Discontinuous Galerkin methods stabilized via the addition of a penalty term defined on the element edges.

\section{NUMERICAL ANALYSIS}

In this section we present a numerical analysis of the LDGC method (42) using the same kind of arguments usually employed in the analysis of discontinuous Galerkin methods for elliptic problems $[4,6]$. Following we define a norm to prove stability and continuity of the bilinear form $A(\cdot, \cdot)$ in the finite dimension product space $V_{h} \times M_{h}$.

Stability and continuity of the LDGC method (42) are proved in the norm

$$
\|\{v, \mu\}\|_{\mathcal{G C}}^{2}:=\|v\|_{\mathcal{E}}^{2}+|\mu-\{v\}|_{\#}^{2}, \quad \forall\{v, \mu\} \in V_{h} \times M_{h}
$$

where

$$
|v|_{\#}^{2}:=\sum_{e \in \mathcal{E}_{h}^{0}} h^{-1}\|v\|_{0, e}^{2}
$$

\subsection{Existence and uniqueness}

The Lax-Milgram lemma is used to analyze problem (42). To this end we first prove LDGC stability as follows.

Lemma 4.1. LDGC Stability. There exists $\alpha_{s}>0$ such that

$$
A\left(\left\{v_{h}, \mu_{h}\right\},\left\{v_{h}, \mu_{h}\right\}\right) \geq \alpha_{s}\left\|\left\{v_{h}, \mu_{h}\right\}\right\|_{\mathcal{G C}}^{2}, \quad \forall\left\{v_{h}, \mu_{h}\right\} \in V_{h} \times M_{h} .
$$

\section{Proof:}

See [6] for details.

Lemma 4.2. (Continuity of $A(\cdot, \cdot)$ and $F(\cdot))$ There exist constants $\alpha_{b}<\infty$ and $\gamma_{b}<\infty$ such that

$$
A(\{u, \lambda\},\{v, \mu\}) \leq \alpha_{b}\|\{u, \lambda\}\|_{\mathcal{G C}}\|\{v, \mu\}\|_{\mathcal{G C}} \quad \forall\{v, \mu\} \in V_{h} \times M_{h}
$$

and

$$
F\left(\left\{v_{h}, \mu_{h}\right\}\right) \leq \gamma_{b}\left\|\left\{v_{h}, \mu_{h}\right\}\right\|_{\mathcal{G C}} \quad \forall\{v, \mu\} \in V_{h} \times M_{h}
$$

Proof:

See [6] for details. 


\subsection{Error estimates}

The Theorem 1 summarizes the main results of the numerical analysis of LDGC method.

Theorem 1. The LDGC method (42) has a unique solution $\left\{u_{h}, \lambda_{h}\right\} \in V_{h} \times M_{h}$ and if the exact solution of our model problem (1) is $u \in H^{k+1}(\Omega)$, the following estimates hold for $k \geq l$

$$
\left\|\left\{u-u_{h}, \lambda-\lambda_{h}\right\}\right\|_{\mathcal{G C}} \leq C\left(h^{\min (k+1, r)-1}|||u| \|_{H^{r}\left(\mathcal{T}_{h}\right)}+h^{k}|u|_{k+1, \Omega}+h^{l}|u|_{l+1, \Omega}\right)
$$

and for $k \geq l$ and $s=-1$ (adjoint consistent formulation)

$$
\left\|u-u_{h}\right\|_{0, \Omega} \leq C\left(h^{\min (k+1, r)}|||u| \|_{H^{r}\left(\mathcal{T}_{h}\right)}+h^{k+1}|u|_{k+1, \Omega}+h^{l+1}|u|_{l+1, \Omega}\right) .
$$

\section{Proof:}

Existence and uniqueness of solution are direct consequences of Lemmas 4.1 and 4.2 and the Lax-Milgram lemma. Estimates (53) and (54) were obtained using consistency, adjoint consistency, stability and boundedness of the LDGC formulation.

\section{NUMERICAL RESULTS}

Simple elliptic problems with known exact solutions were utilized in convergence studies in order to observe the behavior of LDGC method in comparison with continuous and discontinuous Galerkin methods. In all examples we numerically evaluate the convergence properties of the proposed methodology aiming at confirming the rates of convergence predicted by the numerical analysis presented in previous section.

\subsection{D Convergence Study}

First we consider the one-dimensional case of problem (1) defined in $\Omega=(0,1)$, with $\varepsilon=1$ and $\sigma=10$. The Dirichlet boundary conditions and the source term chosen according to the smooth exact solution

$$
u(x)=(1-x) e^{-x^{2}} .
$$

We compare the convergence behaviors of the approximations obtained with continuous Galerkin, discontinuous Galerkin and LDGC $(s=-1,0,1)$ methods, using uniform partitions of the domain $(0,1)$ with $8,16,32$ and 64 linear two node elements. In all cases the LDGC global systems have the same number of degrees-of-freedom as the corresponding continuous Galerkin approximations while DG approximations lead to twice this number. In Figure 1(a) and (d) we can observe the convergence rates of $\operatorname{LDGC}(k)$, for $s=-1$ and $k=1,2,3$, compared with continuous Galerkin and SIPG [25] approximations in $L^{2}(0,1)$ and $H^{1}(0,1)$ norms, respectively. For $k=1$, LDGC(1) shows the optimal rates of convergence in both $H^{1}(0,1) O(h)$ and $L^{2}(0,1) O\left(h^{2}\right)$ norms with accuracy identical or even better than the corresponding continuous and discontinuous Galerkin approximations. Much more accurate solutions with reduced computational cost compared to discontinuous Galerkin 


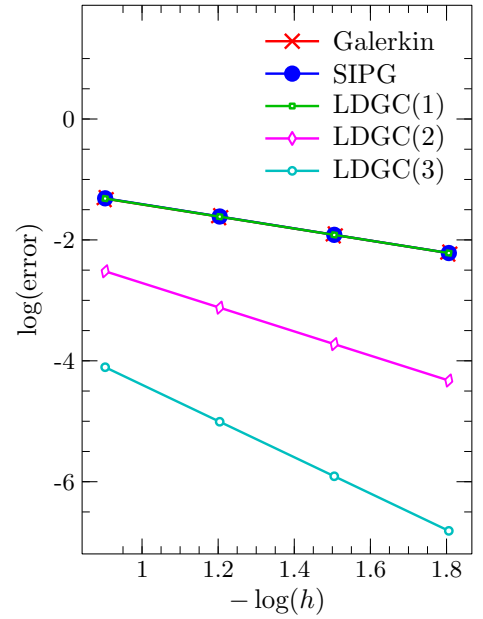

(a) $H^{1}(\Omega)-s=-1$.

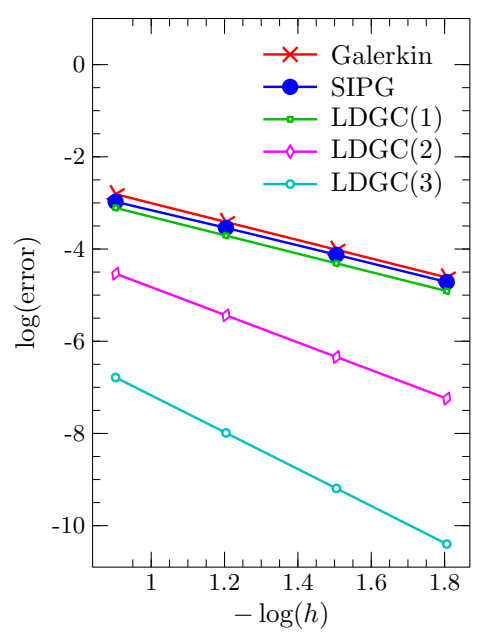

(d) $L^{2}(\Omega)-s=-1$.

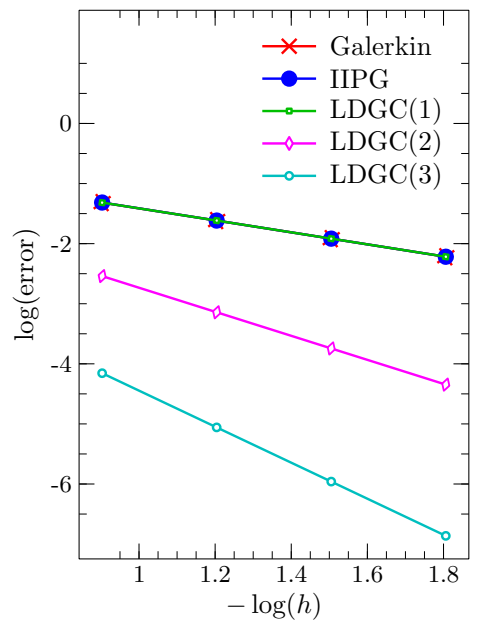

(b) $H^{1}(\Omega)-s=0$.

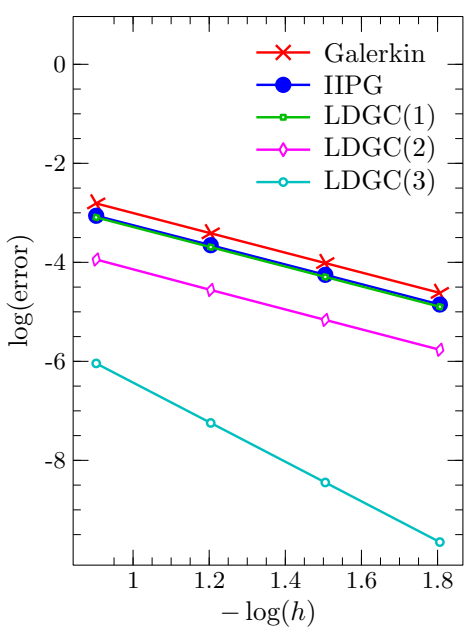

(e) $L^{2}(\Omega)-s=0$.

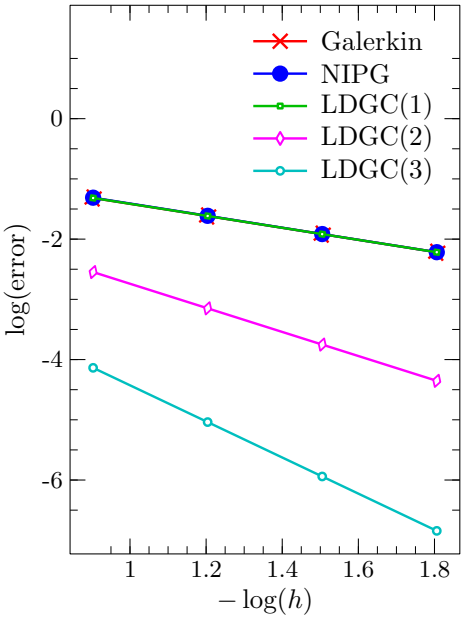

(c) $H^{1}(\Omega)-s=1$.

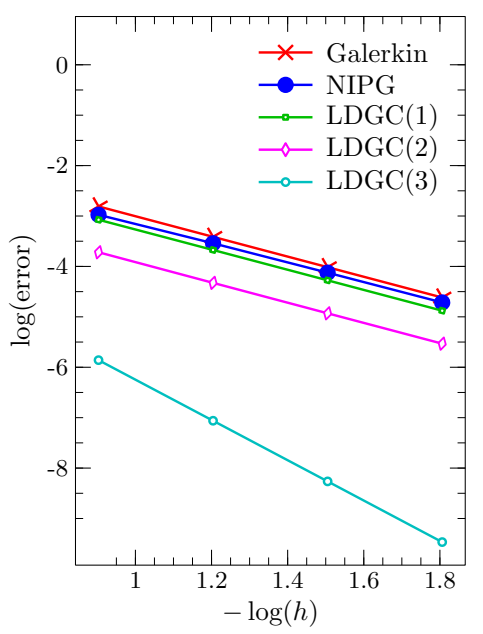

(f) $L^{2}(\Omega)-s=1$.

Figure 1. 1D Convergence rates; $s=-1,0,1 ; \varepsilon=1$ and $\sigma=10$. 
approximations are obtained with LDGC(2) and LDGC(3) as also shown in Figures 1(a) and (d). As predicted in the numerical analysis, optimal rates of convergence are observed for both LDGC(2) $\left(O\left(h^{2}\right)\right.$ in $H^{1}(0,1), O\left(h^{3}\right)$ in $\left.L^{2}(0,1)\right)$ and LDGC(3) $\left(O\left(h^{3}\right)\right.$ in $H^{1}(0,1), O\left(h^{4}\right)$ in $\left.L^{2}(0,1)\right)$ with $s=-1$. Similar results are presented for $s=0$ in Figure 1(b), (e) and for $s=1$ in Figure 1(c), (f). In all cases, the LDGC method presents optimal rates of convergence in $H^{1}(0,1)$ norm. For $s \neq-1$, the DG formulations loose adjoint consistency and their observed convergence rates in $L^{2}(0,1)$ are, as expected, $O\left(h^{k+1}\right)$ if $k$ is odd and $O\left(h^{k}\right)$ if $k$ is even. We use $\beta_{0}=6$ for $k=1, \beta_{0}=12$ for $k=2, \beta_{0}=24$ for $k=3$.

\subsection{D Convergence Study}

We now consider problem (1) defined in the two dimension domain $\Omega=(0,1) \times(0,1)$ with $\varepsilon=1$ and $\sigma=10$. The source term and the Dirichlet boundary conditions chosen according to the exact solution

$$
u(x, y)=\sin (\pi x) \sin (\pi y) .
$$

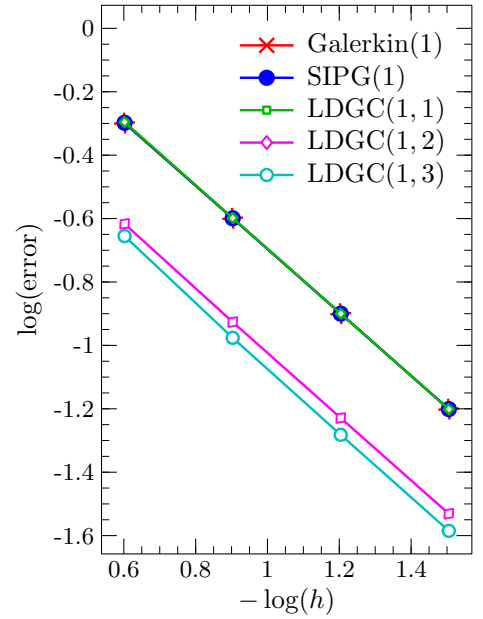

(a) $H^{1}(\Omega)-s=-1$.

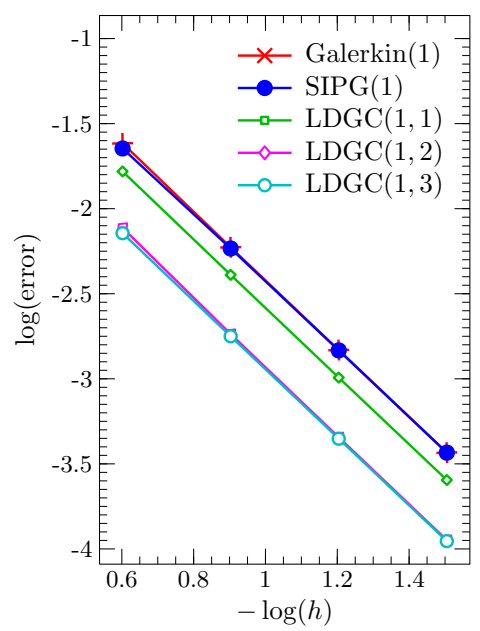

(d) $L^{2}(\Omega)-s=-1$.

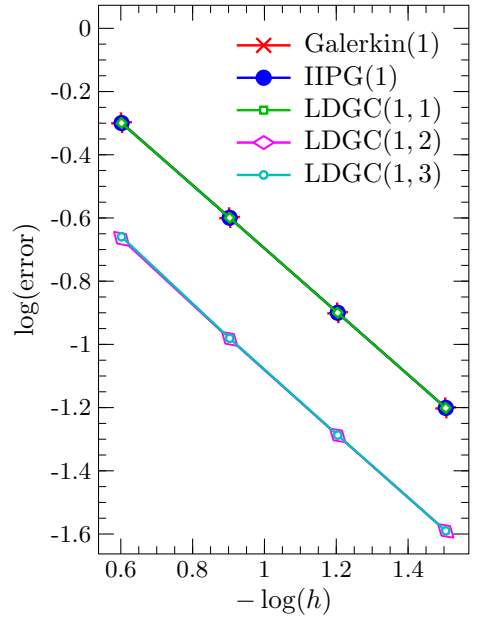

(b) $H^{1}(\Omega)-s=0$.

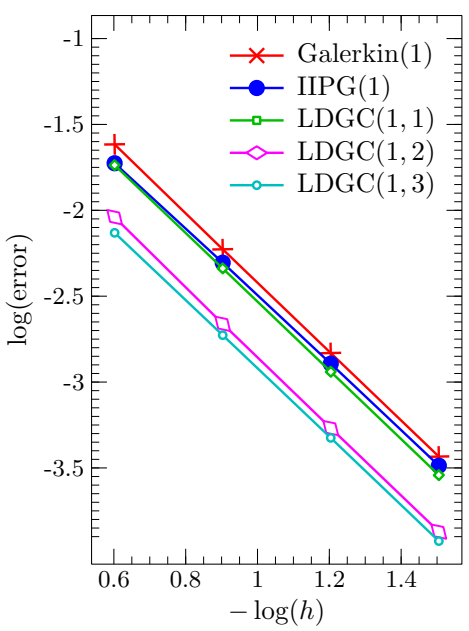

(e) $L^{2}(\Omega)-s=0$.

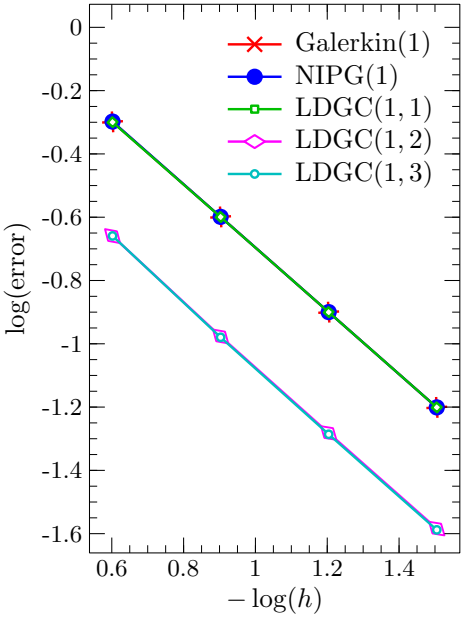

(c) $H^{1}(\Omega)-s=1$.

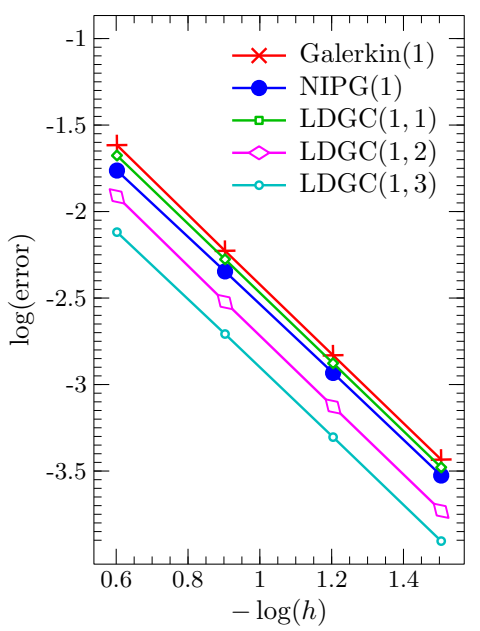

(f) $L^{2}(\Omega)-s=1$.

Figure 2. 2D Convergence rates; $s=-1,0,1 ; k=l=1$; Quadrilateral Elements. 
The LDGC solutions will be identified by $\operatorname{LDGC}(l, k)$ in which $l \geq 1$ is the degree of the polynomial interpolations of the multiplier $\lambda_{h}$ on the element edges and $k$ is the degree of polynomial interpolation of the primal variable $u_{h}$ in the interior of each element. Figure 2 presents a comparison between convergence rates obtained with the Galerkin, DG, and LDGC methods. In these studies we use uniform partitions of the domain with 16, 64, 256 and 1024 quadrilateral elements. We present results of convergence for LDGC method for $s=-1,0,1$, setting $l=1$ and $k=1,2,3$. Unlike the $1 \mathrm{D}$ case, in this $2 \mathrm{D}$ example the rates of convergence of $\operatorname{LDGC}(l, k)$ are associated to the degree $(l=1)$ of the interpolation polynomial adopted for the multiplier for $\lambda_{h}$, as presented in numerical analysis (see Theorem 1). Nevertheless, $\operatorname{LDGC}(1,2)$ and $\operatorname{LDGC}(1,3)$ approximations are more accurate than $\operatorname{LDGC}(1,1)$ and continuous or discontinuous Galerkin approximations in both $L^{2}(\Omega)$ and $H^{1}(\Omega)$ norms.

\subsection{Strongly Reactive Problem}

We now consider the problem (1) defined in the two dimension domain $\Omega=(0,1) \times$ $(0,1)$ with $\varepsilon=10^{-4}, \sigma=1$ and the source term $f=1$. The Dirichlet boundary conditions are homogeneous in $\Gamma$.

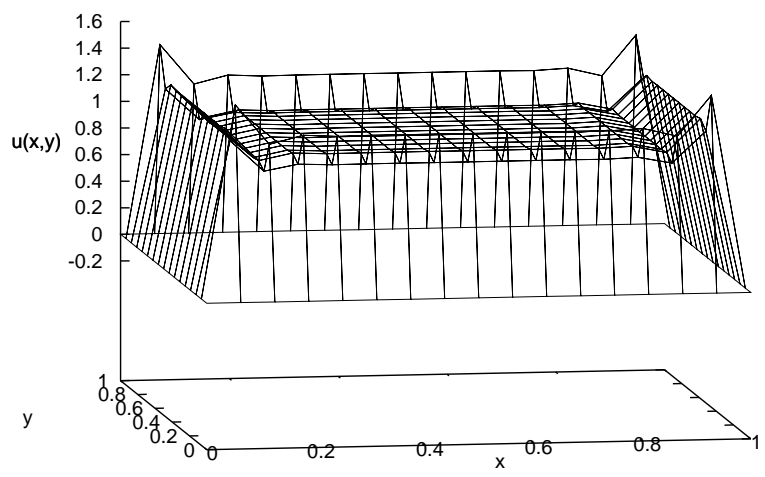

(a) Galerkin method.

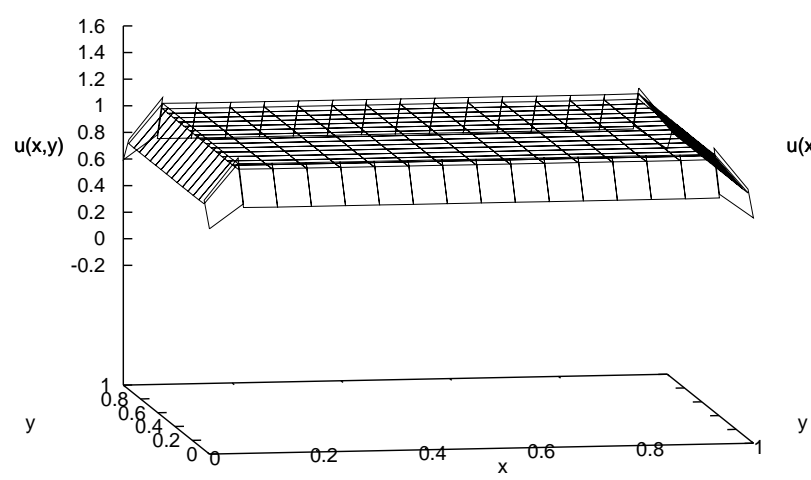

(b) SIPG method.

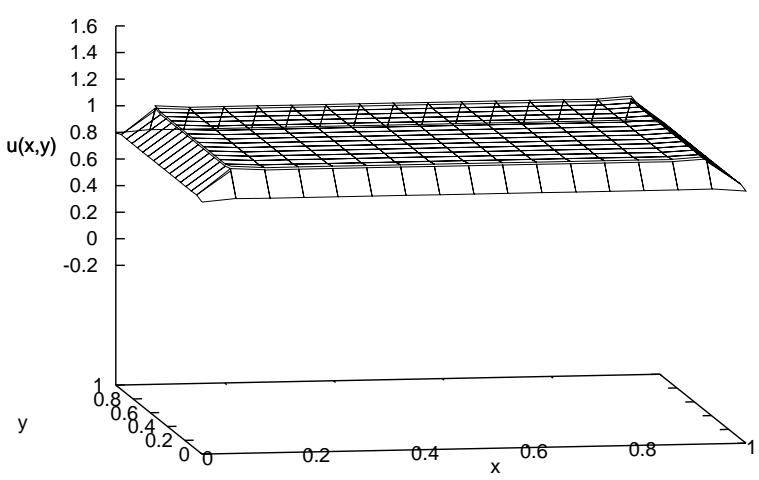

(c) LDGC method.

Figure 3. Strongly Reactive Problem; $s=-1 ; k=l=1 ; 256$ elements. 
Figure 3 compare the approximate solutions obtained with Galerkin, SIPG and LDGC methods. We set $\beta_{0}=2$ for LDGC method and $k=l=1$ for all methods evaluated, using an uniform partition of the domain with 256 elements. Figure 3(a) shows the Galerkin method approximate solution, which presents spurious oscillations for this problem. These oscillations are reduced using the SIPG method as shown in Figure 3(b). However, the LDGC solution is more accurate than Galerkin and SIPG methods, as depicted in Figure 3(c), where these oscillations are elliminated.

\section{CONCLUDING REMARKS}

A Locally Discontinuous but Globally Continuous (LDGC) finite element formulation for reaction-diffusion problems is proposed. The LDGC method is developed from a stabilized hybrid formulation consisting of a set of local problems defined at the element level and a global problem associated with the multiplier. The LDGC method uses the same data structure of continuous Galerkin finite element methods, allowing different degrees of interpolation polynomials for the primal variable and for the multiplier, resulting in more flexibility and improved accuracy. The numerical analysis of LDGC method was presented, attesting that it preserves the main properties of DG methods such as consistency, local conservation, stability, boundedness and optimal rates of convergence in the energy norm, and in $L^{2}(\Omega)$ norm for adjoint consistent formulations.

Some academic numerical experiments are conducted to illustrate the behavior of the LDGC method applied to regular reaction-diffusion problems confirming the optimal rates of convergence predicted by the numerical analysis. Numerical experiments with a $1 \mathrm{D}$ problem confirmed the optimal rates of convergence predicted in the numerical analysis for the two node element with any degree $k \geq 1$ of the interpolation polynomial adopted for the discontinuous field. For 2D problems, optimal rates of convergence are proved and numerically confirmed for $k=l$, with $l$ denoting the degree of the polynomial interpolation adopted for the multiplier. For $k>l$ we have observed improved accuracy of the LDGC approximations compared with continuous and discontinuous Galerkin approximations with the same number of elements in both $H^{1}(\Omega)$ seminorm and $L^{2}(\Omega)$ norm. For strongly reactive problems the LDGC method presents accurate approximate solutions, avoiding spurious oscillations. In all examples analyzed the LDGC approximations exhibited accuracy equivalent or even better than the corresponding continuous or discontinuous Galerkin approximations.

\section{Acknowledgements}

This research is partially supported by the Brazilian Government, through the Agency CNPq, contracts 150994/2010-9, 352991/1992-5 and 304150/2010-0.

\section{REFERENCES}

[1] S. Adjerid, K. D. Devine, J. E. Flaherty, and L. Krivodonova. A posteriori error estimation for discontinuous Galerkin solutions of hyperbolic problems. Computer Methods in Applied Mechanics and Engineering, 191(11-12):1097 - 1112, 2002. 
[2] D. N. Arnold. An interior penalty finite element method with discontinuous elements. SIAM J. Numer. Anal., 19(4):742-760, 1982.

[3] D. N. Arnold, F. Brezzi, B. Cockburn, and D. Marini. Discontinuous Galerkin methods for elliptic problems. In Discontinuous Galerkin methods (Newport, RI, 1999), volume 11 of Lect. Notes Comput. Sci. Eng., pages 89-101. Springer, Berlin, 2000.

[4] D. N. Arnold, F. Brezzi, B. Cockburn, and L. D. Marini. Unified analysis of discontinuous Galerkin methods for elliptic problems. SIAM J. Numer. Anal., 39(5):1749-1779, 2001/02.

[5] N. C. Arruda, R. C. Almeida, and E. G. D. do Carmo. Discontinuous subgrid formulations for transport problems. Computer Methods in Applied Mechanics and Engineering, 199(49-52):3227 - 3236, 2010.

[6] N. C. Arruda, A. F. D. Loula, and R. C. Almeida. Locally continuous but globally continuous galerkin methods for elliptic problems. (submitted).

[7] C. E. Baumann and J. T. Oden. A discontinuous $h p$ finite element method for convectiondiffusion problems. Comput. Methods Appl. Mech. Engrg., 175(3-4):311-341, 1999.

[8] F. Brezzi, B. Cockburn, L. D. Marini, and E. Süli. Stabilization mechanisms in discontinuous Galerkin finite element methods. Comput. Methods Appl. Mech. Engrg., 195(25-28):3293-3310, 2006.

[9] A. Buffa, T. J. R. Hughes, and G. Sangalli. Analysis of a multiscale discontinuous Galerkin method for convection-diffusion problems. SIAM J. Numer. Anal., 44(4):14201440 (electronic), 2006.

[10] B. Cockburn, B. Dong, and J. Guzmán. A superconvergent LDG-hybridizable Galerkin method for second-order elliptic problems. Math. Comp., 77(264):1887-1916, 2008.

[11] B. Cockburn, B. Dong, J. Guzmán, M. Restelli, and R. Sacco. A hybridizable discontinuous Galerkin method for steady-state convection-diffusion-reaction problems. SIAM J. Sci. Comput., 31(5):3827-3846, 2009.

[12] B. Cockburn, J. Gopalakrishnan, and R. Lazarov. Unified hybridization of discontinuous galerkin, mixed, and continuous galerkin methods for second order elliptic problems. 47(2):1319-1365, 2009.

[13] B. Cockburn, J. Gopalakrishnan, and F.-J. Sayas. A projection-based error analysis of HDG methods. Math. Comp., 79(271):1351-1367, 2010.

[14] E. G. D. do Carmo and A. V. C. Duarte. New formulations and numerical analysis of discontinuous Galerkin methods. Comput. Appl. Math., 21(3):661-715, 2002.

[15] E. G. Dutra do Carmo and A. V. C. Duarte. A discontinuous finite element-based domain decomposition method. Comput. Methods Appl. Mech. Engrg., 190(8-10):825$843,2000$. 
[16] P. Houston, C. Schwab, and E. Süli. Discontinuous hp-finite element methods for advection-diffusion-reaction problems. SIAM J. Numer. Anal., 39(6):2133-2163, 2002.

[17] T. J. R. Hughes, G. Scovazzi, P. B. Bochev, and A. Buffa. A multiscale discontinuous Galerkin method with the computational structure of a continuous Galerkin method. Comput. Methods Appl. Mech. Engrg., 195(19-22):2761-2787, 2006.

[18] C. Johnson, U. Nävert, and J. Pitkäranta. Finite element methods for linear hyperbolic problems. Comput. Methods Appl. Mech. Engrg., 45(1-3):285-312, 1984.

[19] C. Johnson and J. Pitkäranta. An analysis of the discontinuous Galerkin method for a scalar hyperbolic equation. Math. Comp., 46(173):1-26, 1986.

[20] S. Kaya and B. Rivière. A discontinuous subgrid eddy viscosity method for the timedependent Navier-Stokes equations. SIAM J. Numer. Anal., 43(4):1572-1595 (electronic), 2005.

[21] W. Klieber and B. Rivière. Adaptive simulations of two-phase flow by discontinuous galerkin methods. Comput. Methods Appl. Mech. Engrg., 196:404-419, 2006.

[22] P. Lesaint and P.-A. Raviart. On a finite element method for solving the neutron transport equation. In Mathematical aspects of finite elements in partial differential equations (Proc. Sympos., Math. Res. Center, Univ. Wisconsin, Madison, Wis., 1974), pages 89123. Publication No. 33. Math. Res. Center, Univ. of Wisconsin-Madison, Academic Press, New York, 1974.

[23] N. Nguyen, J. Peraire, and B. Cockburn. An implicit high-order hybridizable discontinuous galerkin method for nonlinear convection-diffusion equations. Journal of Computational Physics, 228(23):8841 - 8855, 2009.

[24] W. H. Reed and T. R. Hill. Triangular mesh methods for the neutron transport equation. Technical Report LA-UR-73-479, Los Alamos Scientific Laboratory, 1973.

[25] B. Rivière. Discontinuous Galerkin Methods for Solving Elliptic and Parabolic Equations: theory and implementation. SIAM, 2008.

[26] F. A. Rochinha, G. B. Alvarez, E. G. D. do Carmo, and A. F. D. Loula. A locally discontinuous enriched finite element formulation for acoustics. Comm. Numer. Methods Engrg., 23(6):623-637, 2007.

[27] S. Sun and M. F. Wheeler. Symmetric and nonsymmetric discontinuous Galerkin methods for reactive transport in porous media. SIAM J. Numer. Anal., 43(1):195-219, 2005. 